\title{
Rigorous theoretical constraint on constant negative EoS parameter $\omega$ and its effect for the late Universe
}

\author{
Alvina Burgazli ${ }^{1, \mathrm{a}}$, Maxim Eingorn ${ }^{2,3,4, \mathrm{~b}}$, Alexander Zhuk ${ }^{2, \mathrm{c}}$ \\ ${ }^{1}$ Department of Theoretical Physics, Odessa National University, Dvoryanskaya st. 2, Odessa 65082, Ukraine \\ 2 Astronomical Observatory, Odessa National University, Dvoryanskaya st. 2, Odessa 65082, Ukraine \\ ${ }^{3}$ Department of Theoretical and Experimental Nuclear Physics, Odessa National Polytechnic University, Shevchenko av. 1, \\ Odessa 65044, Ukraine \\ ${ }^{4}$ CREST and NASA Research Centers, North Carolina Central University, Fayetteville st. 1801, Durham, NC 27707, USA
}

Received: 4 April 2014 / Accepted: 26 February 2015 / Published online: 12 March 2015

(C) The Author(s) 2015. This article is published with open access at Springerlink.com

\begin{abstract}
In this paper, we consider the Universe at the late stage of its evolution and deep inside the cell of uniformity. At these scales, the Universe is filled with inhomogeneously distributed discrete structures (galaxies, groups and clusters of galaxies). Supposing that the Universe contains also the cosmological constant and a perfect fluid with a negative constant equation of state (EoS) parameter $\omega$ (e.g., quintessence, phantom or frustrated network of topological defects), we investigate scalar perturbations of the Friedmann-Robertson-Walker metrics due to inhomogeneities. Our analysis shows that, to be compatible with the theory of scalar perturbations, this perfect fluid, first, should be clustered and, second, should have the EoS parameter $\omega=-1 / 3$. In particular, this value corresponds to the frustrated network of cosmic strings. Therefore, the frustrated network of domain walls with $\omega=-2 / 3$ is ruled out. A perfect fluid with $\omega=-1 / 3$ neither accelerates nor decelerates the Universe. We also obtain the equation for the nonrelativistic gravitational potential created by a system of inhomogeneities. Due to the perfect fluid with $\omega=-1 / 3$, the physically reasonable solutions take place for flat, open and closed Universes. This perfect fluid is concentrated around the inhomogeneities and results in screening of the gravitational potential.
\end{abstract}

\section{Introduction}

The accelerated expansion of the Universe at late stages of its evolution, found little more than a decade ago $[1,2]$, is

\footnotetext{
a e-mail: aburgazli@gmail.com

b e-mail: maxim.eingorn@gmail.com

c e-mail: ai.zhuk2@gmail.com
}

one of the most intriguing puzzles of modern physics and cosmology. Recognition of this fact was the awarding of the Nobel Prize in 2011 to Saul Perlmutter, Adam Riess and Brian Schmidt. After their discovery, there were numerous attempts to explain the nature of such acceleration. Unfortunately, there is no satisfactory explanation so far (see, e.g., the state of art in [3]). According to the recent observations [4-6], the $\Lambda \mathrm{CDM}$ model is the preferable one. Here, the accelerated expansion is due to the cosmological constant. However, there is a number of problems associated with the cosmological constant. Maybe, one of the main of them consists in the adjustment mechanism which could compensate originally huge vacuum energy down to the cosmologically acceptable value and to solve the coincidence problem of close magnitudes of the non-compensated remnants of vacuum energy and the energy density of the Universe at the present time [7]. To resolve this problem, it was proposed to introduce scalar fields as a matter source. Such scalar fields can be equivalently considered in the form of perfect fluids. Among these perfect fluids, a barotropic fluid is one of the most popular objects of study. This fluid is characterized by the pressure which is the function of the energy density only: $p=p(\rho)$, and the linear equation of state $(\operatorname{EoS}) p=\omega \rho$ is the most popular. These barotropic perfect fluids with the EoS parameters $\omega<-1 / 3$ can cause the accelerated expansion of the Universe. Such fluids are called quintessence [8$10]$ and phantom [11,12] for $-1<\omega<0$ and $\omega<-1$, respectively. Usually, they have a time varying parameter $\omega$ of the EoS. However, there is also a possibility to construct models with constant $\omega$ (for the corresponding experimental restrictions see, particularly, Planck 2013 results [6]). This imposes severe restrictions on the form of the scalar field potential $[13,14]$. In this case, a scalar field is equivalent to a perfect fluid with $\omega=$ const. A large class of mod- 
els is expected to be well described (at least as far as the CMB anisotropy is concerned) by an effective constant EoS parameter [15]. For example, it is also well known that frustrated networks of topological defects (cosmic strings and domain walls) have the form of perfect fluids with the constant parameter $\omega[14,16-18]$. For example, $\omega=-1 / 3$ and $\omega=-2 / 3$ for cosmic strings and domain walls, respectively. It is of interest to investigate the viability of the models with constant $\omega$ and to answer the question whether these models are an alternative to the cosmological constant.

In our paper, we consider the compatibility of these models with the scalar perturbations of the Friedmann-RobertsonWalker (FRW) metrics. In the hydrodynamical approach, such investigation was performed in a number of papers (see, e.g. [19] for $\omega=$ const and [20,21] for $\omega \neq$ const). We consider the Universe at late stages of its evolution when galaxies and clusters of galaxies have already formed. At scales much larger than the characteristic distance between these inhomogeneities, the Universe is well described by the homogeneous and isotropic FRW metrics. This is approximately $190 \mathrm{Mpc}$ and larger [22]. At these scales, the matter fields (e.g., cold dark matter) are well described by the hydrodynamical approach. However, at smaller scales the Universe is highly inhomogeneous. Here, the mechanical approach looks more adequate [22,23]. It is worth noting that similar ideas concerning the discrete cosmology have been discussed in the recent papers $[24,25]$. Obviously, at early stages of the Universe evolution (i.e. before the inhomogeneities formation when the density contrast is much less than unity), the hydrodynamical approach works very well at small scales. It is clear that cosmological models should be tested at all stages of the Universe evolution. It is not sufficient to show their compatibility with observations only at early stages, i.e. in the hydrodynamical approach, as in previous papers. These models should also be in agreement with the mechanical approach. This is the aim and the novelty of our study. To start with, in the present paper we consider the simplest model where a perfect fluid has a constant parameter of the EoS. This article belongs to a series of studies where we intend to test different cosmological models for their compatibility with the mechanical approach. Recently, such investigation was performed for nonlinear $f(R)$ models [26] as well as models with quark-gluon nuggets [27]. In the following paper we will consider the case of time-dependent parameters of the EoS.

In mechanical approach, galaxies, dwarf galaxies and clusters of galaxies (all of them mostly composed of dark matter) can be considered as separate compact objects. Moreover, at distances much greater than their characteristic sizes they can be well described as point-like matter sources. This is generalization of the well-known astrophysical approach
[28] (see \$106) to the case of dynamical cosmological background. Usually, gravitational fields of these inhomogeneities are weak and their peculiar velocities are much less than the speed of light. Therefore, we can construct a theory of perturbations where the considered point-like inhomogeneities disturb the FRW metrics. Such theory was proposed in the paper [23]. Then we applied this mechanical approach in [29] to describe the mutual motion of galaxies, in particular, the Milky Way and Andromeda galaxies. For such investigations, the form of the gravitational potential plays an important role. Hence, one of the main tasks of the present paper is to study a possibility to get a reasonable form of gravitational potentials in the models with an additional perfect fluid with constant negative $\omega$. Then, if such potentials exist, we can study the relative motion of galaxies in the field of these potentials and compare it with the corresponding motion in the $\Lambda$ CDM model [29].

Because perfect fluids have $\omega=$ const, their perturbations are purely adiabatic (see, e.g. [30]), i.e. dissipative processes are absent. Then we demonstrate that, first, these fluids must be clustered (i.e. inhomogeneous) and, second, $\omega=-1 / 3$ is the only parameter which is compatible with the theory of scalar perturbations. It is well known that such perfect fluid neither accelerates nor decelerates the Universe. Frustrated network of cosmic strings is a possible candidate for such perfect fluid. It is worth noting that this conclusion is valid for perfect fluids with the constant EoS parameter. The conclusion for imperfect fluids (e.g., for scalar fields with arbitrary potentials) can be quite different. We also obtain formulas for the nonrelativistic gravitational potential created by a system of inhomogeneities (galaxies, groups, and clusters of galaxies). We show that due to the perfect fluid with $\omega=-1 / 3$, the physically reasonable expressions take place for flat, open, and closed Universes. If such perfect fluid is absent, the hyperbolic space is preferred [23]. Hence, even if this perfect fluid does not accelerate the Universe, it can play an important role. It is worth noting also that according to the paper [18], a small contribution from the string network can explain the possible small departure from $\Lambda \mathrm{CDM}$ evolution.

The paper is structured as follows. In Sect. 2, we consider scalar perturbations in the Friedmann Universe filled with the cosmological constant, pressureless dustlike matter (baryon and dark matter) and perfect fluid with negative constant EoS. Here, we get the equation for the nonrelativistic gravitational potential. In Sect. 3, we find solutions of this equation for an arbitrary system of inhomogeneities for flat, open, and closed Universes. These solutions have the Newtonian limit in the vicinity of inhomogeneities and are finite at any point outside inhomogeneities. The main results are summarized in the concluding Sect. 4. 


\section{Scalar perturbations of FRW Universe}

\subsection{Homogeneous background}

To start with, we consider a homogeneous isotropic Universe described by the FRW metrics,

$$
\begin{aligned}
\mathrm{d} s^{2} & =a^{2}\left(\mathrm{~d} \eta^{2}-\gamma_{\alpha \beta} \mathrm{d} x^{\alpha} \mathrm{d} x^{\beta}\right) \\
& =a^{2}\left(\mathrm{~d} \eta^{2}-\mathrm{d} \chi^{2}-\Sigma^{2}(\chi) \mathrm{d} \Omega_{2}^{2}\right),
\end{aligned}
$$

where

$$
\Sigma(\chi)= \begin{cases}\sin \chi, & \chi \in[0, \pi] \text { for } \mathcal{K}=+1 \\ \chi, & \chi \in[0,+\infty) \text { for } \mathcal{K}=0 \\ \sinh \chi, & \chi \in[0,+\infty) \text { for } \mathcal{K}=-1\end{cases}
$$

and $\mathcal{K}=-1,0,+1$ for open, flat, and closed Universes, respectively. As matter sources, we consider the cosmological constant ${ }^{1} \Lambda$, pressureless dustlike matter (in accordance with the current observations [4,5], we assume that dark matter (DM) gives the main contribution to this matter) and an additional perfect fluid with the $\operatorname{EoS} \bar{p}=\omega \bar{\varepsilon}$ where $\omega<0$. In the present paper, $\omega=$ const. As we already wrote in the Introduction, such perfect fluids can be modeled by scalar fields with the corresponding form of the potentials $[13,14]$ as well as by the frustrated network of the topological defects $[14,16-18]$. We exclude the values $\omega=0,-1$ because they are equivalent to DM and the cosmological constant, respectively. Scalar fields with $-1<\omega<0$ and $\omega<-1$ are usually called quintessence and phantom, respectively. Below, the overline denotes homogeneous perfect fluids. It can easily be seen from the conservation equation that in the case of the homogeneous perfect fluid

$\bar{\varepsilon}=\varepsilon_{0} \frac{a_{0}^{3(1+\omega)}}{a^{3(1+\omega)}}$,

where $a_{0}$ is the scale factor at the present time and $\varepsilon_{0}$ is the current value of the energy density $\bar{\varepsilon}$.

Because we consider the late stages of the Universe evolution, we neglect the contribution of radiation. It is worth noting that radiation can also be included into consideration [22], and the simple analysis demonstrates that this does not affect the results of the paper. Therefore, the Friedmann

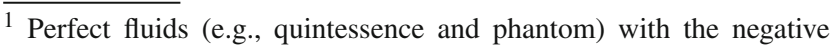
parameter of the EoS $\omega<-1 / 3$ were introduced to explain the late time acceleration of the Universe. They are an alternative to the cosmological constant. However, in our model, we shall keep both perfect fluids and the cosmological constant because we investigate the full range of negative parameters $\omega<0$. Moreover, we shall show that the only possible value of $\omega$ for the considered perfect fluid is $-1 / 3$. Then the inclusion of $\Lambda$ becomes justified. Additionally, a small contribution from these fluids (e.g., frustrated network of cosmic strings with $\omega=-1 / 3$ ) can explain the possible small departure from $\Lambda$ CDM evolution [18].
}

equations read

$$
\frac{3\left(\mathcal{H}^{2}+\mathcal{K}\right)}{a^{2}}=\kappa \bar{T}_{0}^{0}+\Lambda+\kappa \bar{\varepsilon}
$$

and

$$
\frac{2 \mathcal{H}^{\prime}+\mathcal{H}^{2}+\mathcal{K}}{a^{2}}=\Lambda-\kappa \omega \bar{\varepsilon}
$$

where $\mathcal{H} \equiv a^{\prime} / a \equiv(\mathrm{d} a / \mathrm{d} \eta) / a$ and $\kappa \equiv 8 \pi G_{\mathrm{N}} / c^{4}$ ( $c$ is the speed of light and $G_{\mathrm{N}}$ is the Newton gravitational constant). Here, $\bar{T}^{i k}$ is the energy-momentum tensor of the average pressureless dustlike matter. For such matter, the energy density $\bar{T}_{0}^{0}=\bar{\rho} c^{2} / a^{3}$ is the only nonzero component. $\bar{\rho}=$ const is the comoving average rest mass density [23]. It is worth noting that in the case $\mathcal{K}=0$ the comoving coordinates $x^{\alpha}$ may have a dimension of length, but then the conformal factor $a$ is dimensionless, and vice versa. However, in the cases $\mathcal{K}= \pm 1$ the dimension of $a$ is fixed. Here, $a$ has a dimension of length and $x^{\alpha}$ are dimensionless. For consistency, we shall follow this definition for $\mathcal{K}=0$ as well. For such choice of the dimension of $a$, the rest mass density has a dimension of mass.

Conformal time $\eta$ and synchronous time $t$ are connected as $c \mathrm{~d} t=a \mathrm{~d} \eta$. Therefore, Eqs. (2.4) and (2.5), respectively, take the form

$$
H^{2}=H_{0}^{2}\left(\Omega_{M} \frac{a_{0}^{3}}{a^{3}}+\Omega_{\Lambda}+\Omega_{\mathcal{K}} \frac{a_{0}^{2}}{a^{2}}+\Omega_{\mathrm{pf}} \frac{a_{0}^{3(1+\omega)}}{a^{3(1+\omega)}}\right)
$$

and

$$
\frac{\ddot{a}}{a}=H_{0}^{2}\left(-\frac{1}{2} \Omega_{M} \frac{a_{0}^{3}}{a^{3}}+\Omega_{\Lambda}-\frac{1}{2}(1+3 \omega) \Omega_{\mathrm{pf}} \frac{a_{0}^{3(1+\omega)}}{a^{3(1+\omega)}}\right),
$$

where $a_{0}$ and $H_{0}$ are the values of the conformal factor $a$ and the Hubble "constant" $H \equiv \dot{a} / a \equiv(\mathrm{d} a / \mathrm{d} t) / a$ at the present time $t=t_{0}$, and we introduced the density parameters:

$$
\begin{aligned}
& \Omega_{M}=\frac{\kappa \bar{\rho} c^{4}}{3 H_{0}^{2} a_{0}^{3}}, \quad \Omega_{\Lambda}=\frac{\Lambda c^{2}}{3 H_{0}^{2}}, \\
& \Omega_{\mathcal{K}}=-\frac{\mathcal{K} c^{2}}{a_{0}^{2} H_{0}^{2}}, \quad \Omega_{\mathrm{pf}}=\frac{\kappa c^{2} \varepsilon_{0}}{3 H_{0}^{2}},
\end{aligned}
$$

therefore

$\Omega_{M}+\Omega_{\Lambda}+\Omega_{\mathcal{K}}+\Omega_{\mathrm{pf}}=1$.

It is of interest to get the experimental restriction on $\Omega_{\mathrm{pf}}$. This requires a separate investigation which is out of the scope of our paper. We can easily see from Eq. (2.7) that perfect fluids with $\omega<-1 / 3$ can provide the accelerated expansion of the Universe. 


\subsection{Scalar perturbations}

As we have written in the Introduction, the inhomogeneities in the Universe result in scalar perturbations of the metrics (2.1). In the conformal Newtonian gauge, such perturbed metrics is $[31,32]$

$\mathrm{d} s^{2} \approx a^{2}\left[(1+2 \Phi) \mathrm{d} \eta^{2}-(1-2 \Psi) \gamma_{\alpha \beta} \mathrm{d} x^{\alpha} \mathrm{d} x^{\beta}\right]$,

where scalar perturbations $\Phi, \Psi \ll 1$. Following the standard argumentation, we can put $\Phi=\Psi$. We consider the Universe at the late stage of its evolution when the peculiar velocities of inhomogeneities (both for dustlike matter and the considered perfect fluid) are much less than the speed of light:

$\frac{\mathrm{d} x^{\alpha}}{\mathrm{d} \eta}=a \frac{\mathrm{d} x^{\alpha}}{\mathrm{d} t} \frac{1}{c} \equiv \frac{v^{\alpha}}{c} \ll 1$.

We should stress that smallness of the nonrelativistic gravitational potential $\Phi$ and peculiar velocities $v^{\alpha}$ are two independent conditions (e.g., for very light relativistic masses the gravitational potential can still remain small). Under these conditions, the gravitational potential $\Phi$ satisfies the following system of equations (see [23] for details ${ }^{2}$ ):

$\Delta \Phi-3 \mathcal{H}\left(\Phi^{\prime}+\mathcal{H} \Phi\right)+3 \mathcal{K} \Phi=\frac{1}{2} \kappa a^{2} \delta T_{0}^{0}+\frac{1}{2} \kappa a^{2} \delta \varepsilon$,

$\frac{\partial}{\partial x^{\beta}}\left(\Phi^{\prime}+\mathcal{H} \Phi\right)=0$

$\Phi^{\prime \prime}+3 \mathcal{H} \Phi^{\prime}+\left(2 \mathcal{H}^{\prime}+\mathcal{H}^{2}\right) \Phi-\mathcal{K} \Phi=\frac{1}{2} \kappa a^{2} \delta p$,

where the Laplace operator

$\triangle=\frac{1}{\sqrt{\gamma}} \frac{\partial}{\partial x^{\alpha}}\left(\sqrt{\gamma} \gamma^{\alpha \beta} \frac{\partial}{\partial x^{\beta}}\right)$

and $\gamma$ is the determinant of $\gamma_{\alpha \beta}$. Following the reasoning of [23], we took into account that peculiar velocities of inhomogeneities are nonrelativistic, and under the corresponding condition (2.11) the contribution of $\delta T_{\beta}^{0}$ is negligible compared to that of $\delta T_{0}^{0}$ both for dustlike matter and the considered perfect fluid. Really, according to [23], the true rest mass density $\rho$ of usual matter, presented by a sum of delta-functions (see Eq. (3.4) below), is comparable with itself after subtracting the average value $\bar{\rho}$. Consequently, $\delta T_{\beta}^{0} / \delta T_{0}^{0} \sim v^{\beta} / c \ll 1$. Exactly the same strong inequality

\footnotetext{
${ }^{2}$ It is well known that in the hydrodynamic approach, the linear formalism is not applicable to study the formation of galaxies and clusters of galaxies. However, first, we consider the late stage of the Universe evolution when these inhomogeneities were mainly formed. Second, in our mechanical approach, we can use the linear approximation due to the smallness of the gravitational fields and peculiar velocities. Here, the structure of the galaxies can evolve on account of mechanical merger of inhomogeneities.
}

holds true also for the additional perfect fluid under the quite natural assumption that only its fraction of the order $\delta \varepsilon / \varepsilon$ takes part in considerable motion due to interaction between inhomogeneities. In other words, account of $\delta T_{\beta}^{0}$ is beyond the accuracy of the model. This approach is completely consistent with [28] where it is shown that the nonrelativistic gravitational potential is defined by the positions of the inhomogeneities but not by their velocities [see Eq. (106.11) in this book]. In the case of an arbitrary number of dimensions, a similar result was obtained in [33]. On the other hand, the motion of nonrelativistic inhomogeneities is defined by the gravitational potential (see, e.g. [29]). The perturbed matter remains nonrelativistic (pressureless) that results in the condition $\delta T_{\beta}^{\alpha}=0$. For the considered perfect fluid we have $\delta T_{\beta}^{\alpha}=-\delta p \delta_{\beta}^{\alpha}$, and $\delta \varepsilon$ is a fluctuation of the energy density for this perfect fluid. In Eq. (2.12) $\delta T_{0}^{0}$ is related to the fluctuation of the energy density of dustlike matter and has the form [23]:

$\delta T_{0}^{0}=\frac{\delta \rho c^{2}}{a^{3}}+\frac{3 \bar{\rho} c^{2} \Phi}{a^{3}}$,

where $\delta \rho$ is the difference between real and average rest mass densities:

$\delta \rho=\rho-\bar{\rho}$.

From Eq. (2.13) we get

$\Phi(\eta, \mathbf{r})=\frac{\varphi(\mathbf{r})}{c^{2} a(\eta)}$,

where $\varphi(\mathbf{r})$ is a function of all spatial coordinates and we have introduced $c^{2}$ in the denominator for convenience. Below, we shall see that $\varphi(\mathbf{r}) \sim 1 / r$ in the vicinity of an inhomogeneity, and the nonrelativistic gravitational potential $\Phi(\eta, \mathbf{r}) \sim 1 /($ ar $)=1 / R$, where $R=$ ar is the physical distance. Hence, $\Phi$ has the correct Newtonian limit near the inhomogeneities. Substituting the expression (2.18) into Eqs. (2.12) and (2.14), we get the following system of equations:

$$
\begin{aligned}
& \frac{1}{a^{3}}(\Delta \varphi+3 \mathcal{K} \varphi)=\frac{1}{2} \kappa c^{2} \delta T_{0}^{0}+\frac{1}{2} \kappa c^{2} \delta \varepsilon, \\
& \frac{1}{a^{3}}\left(\mathcal{H}^{\prime}-\mathcal{H}^{2}-\mathcal{K}\right) \varphi=\frac{1}{2} \kappa c^{2} \delta p .
\end{aligned}
$$

From the Friedmann equations (2.4) and (2.5) we obtain

$\frac{1}{a^{3}}\left(\mathcal{H}^{\prime}-\mathcal{H}^{2}-\mathcal{K}\right)=\frac{1}{2 a}\left(-\kappa \bar{T}_{0}^{0}-\kappa(1+\omega) \bar{\varepsilon}\right)$.

Then Eq. (2.20) reads

$$
\left(-\kappa \frac{\bar{\rho} c^{2}}{a^{4}}-\kappa(1+\omega) \frac{\varepsilon_{0}}{a_{0}} \frac{a_{0}^{4+3 \omega}}{a^{4+3 \omega}}\right) \varphi=\kappa c^{2} \omega \delta \varepsilon .
$$

It should be noted that we consider the perfect fluids without thermal coupling to any other type of matter. It means, in particular, that evolution of its homogeneous background as well as scalar perturbations occurs adiabatically or, in other 
words, without change of entropy. Therefore, in the case of the constant parameter of the EoS we preserve the same linear $\operatorname{EoS} \delta p=\omega \delta \varepsilon$ with the same constant parameter $\omega$ for the scalar perturbations $\delta p$ and $\delta \varepsilon$ of pressure and energy density, respectively, as for their background values $\bar{p}$ and $\bar{\varepsilon}$ (see, e.g., equations (1) and (2) in [30]). Obviously, imperfect fluids such as scalar fields with arbitrary potentials (which results in time-dependent parameter $\omega$ ) require a different approach [34-37].

Taking into account the expression (2.18), we see that in the right hand side of Eq. (2.16) the second term is proportional to $1 / a^{4}$ and should be dropped because we consider the nonrelativistic matter. ${ }^{3}$ This is the accuracy of our approach, i.e. for the terms of the form of $1 / a^{n}$, we drop ones with $n \geq 4$ and leave terms with $n<4$. Obviously, $4+3 \omega<4$ for $\omega<0$. Hence, we can draw the important conclusion regarding the purely homogeneous non-clustered quintessence/phantom fluids with $\delta p, \delta \varepsilon=0$. For these fluids, we arrive at a contradiction because in Eq. (2.22) the right hand side is equal to zero while the left hand side is nonzero. Therefore, such fluids are forbidden. ${ }^{4}$ The considered perfect fluid (quintessence, phantom or frustrated network of topological defects) should be capable of clustering. In the papers $[10,38]$, it was also pointed out that the quintessence has to be inhomogeneous. For the inhomogeneous perfect fluid we get from Eq. (2.22) that

$\delta \varepsilon=-\frac{1+\omega}{c^{2} \omega} \varepsilon_{0} \frac{a_{0}^{3+3 \omega}}{a^{4+3 \omega}} \varphi$.

Substituting (2.23) into (2.19), we obtain within our accuracy

$$
\begin{aligned}
\frac{1}{a^{3}}(\Delta \varphi+3 \mathcal{K} \varphi) & =\frac{1}{2} \kappa c^{2} \frac{\delta \rho c^{2}}{a^{3}}-\frac{1}{2} \kappa c^{2} \frac{1+\omega}{c^{2} \omega} \varepsilon_{0} \frac{a_{0}^{3+3 \omega}}{a^{4+3 \omega}} \varphi \\
\Rightarrow \Delta \varphi+3 \mathcal{K} \varphi & =\frac{1}{2} \kappa c^{4} \delta \rho-\frac{1+\omega}{2 \omega} \kappa \varepsilon_{0} a_{0}^{2} \frac{a_{0}^{1+3 \omega}}{a^{1+3 \omega}} \varphi .
\end{aligned}
$$

In this equation, all terms except the last one do not depend on time. ${ }^{5}$ Therefore, $\omega=-1 / 3$ is the only possibility to avoid this problem. Hence, we arrive at the following important conclusion. At the late stage of the Universe evolution,

\footnotetext{
${ }^{3}$ Radiation can easily be included in our scheme [22]. The simple analysis shows that this does not change all of the following results.

${ }^{4}$ It can easily be realized that the homogeneous solution $\delta \varepsilon=0, \varphi=0$ is forbidden because it contradicts Eq. (2.19). The point is that the standard matter density perturbations $\delta T_{0}^{0}$ defined in Eq. (2.16) are supposed to be nonzero. In other words, we consider the Universe filled with inhomogeneously distributed galaxies, groups, and clusters of galaxies. The presence of these inhomogeneities results in nonzero perturbations of the 00 component of the corresponding energy-momentum tensor [23].

5 We would like to recall that quantities $\varphi$ and $\delta \rho$ are the comoving ones [23]. Therefore, within the adopted accuracy when both nonrelativistic and weak field limits are applied, they do not depend explicitly on time [22].
}

the considered perfect fluids are compatible with the scalar perturbations only if, first, they are inhomogeneous, and, second, they have the EoS parameter $\omega=-1 / 3$. For example, the frustrated network of cosmic strings can be a candidate for this fluid. On the other hand, frustrated domain walls are ruled out because they have $\omega=-2 / 3$. Equation (2.7) clearly demonstrates that the perfect fluid with $\omega=-1 / 3$ neither accelerates nor decelerates the Universe.

It is worth noting that in our model neither the nonrelativistic gravitational potential $\Phi \sim 1 / a$ nor the perfect fluid density contrast $\delta \varepsilon / \bar{\varepsilon} \sim 1 / a$ diverge with time (with the scale factor $a$ ) in spite of the negative sign of the ratio $\delta p / \delta \varepsilon$ which is often treated as the speed of sound squared. In the papers $[39,40]$ it was shown that such components could be stable if sufficiently rigid. Really, as we shall show below, our perfect fluid is not purely fluid. Its fluctuations are concentrated around the matter/dark matter inhomogeneities (see, e.g., Eq. (3.8)). Obviously, the speed of sound in this case is close to zero. As noted in the paper [41], for the "solid" dark energy, the zero speed of sound is preferable. On the other hand, due to the concentration of fluctuations around the matter/dark matter inhomogeneities, they have velocities of the order of the velocities of matter/dark matter. That is, the condition (2.11) is valid for the perfect fluid in spite of the averaged relativistic $\operatorname{EoS} \bar{p}=\omega \bar{\rho}$.

For $\omega=-1 / 3$, the equation for the gravitational potential and the fluctuation of the energy density of the perfect fluid read, respectively:

$\Delta \varphi+\left(3 \mathcal{K}-\frac{8 \pi G_{\mathrm{N}}}{c^{4}} \varepsilon_{0} a_{0}^{2}\right) \varphi=4 \pi G_{\mathrm{N}}(\rho-\bar{\rho})$

and

$\delta \varepsilon=\frac{2 \varepsilon_{0} a_{0}^{2}}{c^{2} a^{3}} \varphi$.

Naturally, Eq. (2.25) coincides with Eq. (2.27) in [23] in the absence of the perfect fluid (i.e. for $\varepsilon_{0}=0$ ). Moreover, for $\mathcal{K}=0$ and $\varepsilon_{0}=0$, this equation coincides (up to an evident redefinition) with Eq. (7.14) in the well-known book [42] and Eq. (2) for the GADGET-2 [43].

In the next section, we shall investigate Eq. (2.25) depending on the curvature parameter $\mathcal{K}$. We shall show that reasonable expressions of the conformal gravitational potential $\varphi$ exist for any sign of $\mathcal{K}$. This takes place due to the presence of the perfect fluid with $\omega=-1 / 3$. If this fluid is absent, the hyperbolic model $\mathcal{K}=-1$ is preferred [23]. Therefore, the positive role of such perfect fluid is that its presence gives a possibility to consider models for any $\mathcal{K}$.

\section{Gravitational potentials}

It is convenient to rewrite Eq. (2.25) as follows:

$\Delta \phi-\lambda^{2} \phi=4 \pi G_{\mathrm{N}} \rho$, 
where the truncated gravitational potential is

$\phi=\varphi-\frac{4 \pi G_{\mathrm{N}} \bar{\rho}}{\lambda^{2}}, \quad \lambda \neq 0$,

and

$\lambda^{2} \equiv \frac{8 \pi G_{\mathrm{N}}}{c^{4}} \varepsilon_{0} a_{0}^{2}-3 \mathcal{K}=\frac{3 a_{0}^{2} H_{0}^{2}}{c^{2}}\left(\Omega_{\mathcal{K}}+\Omega_{\mathrm{pf}}\right)$.

As we have already mentioned in the Introduction, on scales smaller than the cell of uniformity size and on late stages of evolution, the Universe is filled with inhomogeneously distributed discrete structures (galaxies, groups, and clusters of galaxies) with dark matter concentrated around these structures. Then the rest mass density $\rho$ reads [23]

$\rho=\frac{1}{\sqrt{\gamma}} \sum_{i} m_{i} \delta\left(\mathbf{r}-\mathbf{r}_{i}\right)$

where $m_{i}$ is the mass of $i$ th inhomogeneity. Therefore, Eq. (3.1) satisfies the very important principle of superposition. It is sufficient to solve this equation for one gravitating mass $m_{i}$ and obtain its gravitational potential $\phi_{i}$. The gravitational potential for all system of inhomogeneities is equal to a sum of potentials $\phi_{i}$. It is worth recalling that the operator $\Delta$ is defined by Eq. (2.15). As boundary conditions, we demand that, first, the gravitational potential of a gravitating mass should have the Newtonian limit near this inhomogeneity $\phi_{i} \sim 1 / r$ and, second, this potential should converge at any point of the Universe (except the gravitating mass position).

It seems reasonable to assume also that the total gravitational potential averaged over the whole Universe is equal to zero (see, e.g. [23]):

$\bar{\varphi}=\bar{\phi}+\frac{4 \pi G_{\mathrm{N}} \bar{\rho}}{\lambda^{2}}=0, \quad \bar{\phi}=\sum_{i} \frac{1}{V} \int_{V} \phi_{i} \mathrm{~d} V$,

where $V$ is the volume of the Universe. This demand results in another physically reasonable condition: $\overline{\delta \varepsilon}=0$ (see Eq. (2.26))

\subsection{Flat space: $\mathcal{K}=0$}

In the case $\varepsilon_{0}>0 \rightarrow \lambda^{2}=\frac{8 \pi G_{N}}{c^{4}} \varepsilon_{0} a_{0}^{2}>0$, the solution of (3.1) for a separate mass $m_{i}$ satisfying the mentioned above boundary conditions reads

$\phi_{i}=-\frac{G_{\mathrm{N}} m_{i}}{r} \exp (-\lambda r), \quad \lambda>0,0<r<+\infty$.

It can easily be seen that this truncated potential has the Newtonian limit for $r \rightarrow 0$. This expression shows that the perfect fluid results in the screening of the Newtonian potential. A similar effect for the Coulomb potential takes place in plasma. In our case, the screening originates due to specific nature of the perfect fluid. It is worth mentioning that the exponential screening of the gravitational potential was introduced "by hand" in a number of models to solve the famous Seeliger paradox (see, e.g., the review [44]). In our model, we resolve this paradox in a natural way due to the presence of the specific perfect fluid.

For a many-particle system, the total gravitational potential takes the form

$\varphi=-G_{\mathrm{N}} \sum_{i} \frac{m_{i}}{\left|\mathbf{r}-\mathbf{r}_{i}\right|} \exp \left(-\lambda\left|\mathbf{r}-\mathbf{r}_{i}\right|\right)+\frac{4 \pi G_{\mathrm{N}} \bar{\rho}}{\lambda^{2}}$

Substituting (3.7) into (2.26), we get for the fluctuations of the perfect fluid energy density the following expression:

$\delta \varepsilon=\frac{2 \varepsilon_{0} a_{0}^{2}}{c^{2} a^{3}}\left(-G_{\mathrm{N}} \sum_{i} m_{i} \frac{\exp \left(-\lambda\left|\mathbf{r}-\mathbf{r}_{i}\right|\right)}{\left|\mathbf{r}-\mathbf{r}_{i}\right|}+\frac{c^{4} \bar{\rho}}{2 \varepsilon_{0} a_{0}^{2}}\right)$.

Therefore, we arrive at a physically reasonable conclusion that these fluctuations are concentrated around the matter/dark matter inhomogeneities and the corresponding profile is given by Eq. (3.8).

The averaged value of the $i$ th component of the truncated potential over some finite volume $V_{0}$ is

$$
\begin{aligned}
\bar{\phi}_{i} & =\frac{4 \pi}{V_{0}} \int_{0}^{r_{0}}\left[-G_{\mathrm{N}} m_{i} \frac{\exp (-\lambda r)}{r}\right] r^{2} \mathrm{~d} r \\
& =-\frac{4 \pi G_{\mathrm{N}} m_{i}}{V_{0}}\left[-\frac{\exp \left(-\lambda r_{0}\right)}{\lambda}\left(r_{0}+\frac{1}{\lambda}\right)+\frac{1}{\lambda^{2}}\right] .
\end{aligned}
$$

Then, letting the volume go to infinity $\left(r_{0} \rightarrow+\infty \Rightarrow V_{0} \rightarrow\right.$ $+\infty)$ and taking all gravitating masses, we obtain

$\bar{\phi}=-G_{\mathrm{N}} \bar{\rho} \frac{4 \pi}{\lambda^{2}}$,

where $\bar{\rho}=\lim _{V_{0} \rightarrow+\infty} \sum_{i} m_{i} / V_{0}$. Therefore, the averaged gravitational potential (3.5) is equal to zero: $\bar{\varphi}=0$. Consequently, $\overline{\delta \varepsilon}=0$.

The case $\varepsilon_{0}<0 \Rightarrow \lambda^{2} \equiv-\mu^{2}<0$ is not of interest. Here, we get the expression $\phi_{i}=-\left(G_{\mathrm{N}} m_{i} / r\right) \cos (\mu r)$ which does not have clear physical sense. Additionally, this expression does not allow the procedure of averaging.

\subsection{Spherical space: $\mathcal{K}=+1$}

Let us consider, first, the case $\lambda^{2}=\frac{8 \pi G_{N}}{c^{4}} \varepsilon_{0} a_{0}^{2}-3 \equiv-\mu^{2}<$ 0 . This case is of interest because it allows us to perform the transition to small values of the energy density of the perfect fluid: $\varepsilon_{0} \rightarrow 0$. Here, the solution of (3.1) for a separate mass $m_{i}$ is

$\phi_{i}=-G_{\mathrm{N}} m_{i} \frac{\sin \left[(\pi-\chi) \sqrt{\mu^{2}+1}\right]}{\sin \left(\pi \sqrt{\mu^{2}+1}\right) \sin \chi}, \quad 0<\chi \leq \pi$.

For $\sqrt{\mu^{2}+1} \neq 2,3, \ldots$ (we would recall that $\mu^{2} \neq 0$ ), this formula is finite at any point $\chi \in(0, \pi]$ and has the 
Newtonian limit for $\chi \rightarrow 0$. In the case of absence of the perfect fluid $\varepsilon_{0}=0 \rightarrow \sqrt{\mu^{2}+1}=2$, this expression is divergent at $\chi=\pi$. We demonstrated this fact in our paper [23]. Therefore, the considered perfect fluid gives a possibility to avoid this problem for the models with $\mathcal{K}=+1$. It can easily be verified that for the total system of gravitating masses, the averaged value of the total truncated potential has the form of (3.10) that results in $\bar{\varphi}=0 \Rightarrow \overline{\delta \varepsilon}=0$.

In the case $\lambda^{2}>0$, the formulas can easily be found from (3.11) with the help of analytical continuation $\mu \rightarrow i \mu$. In other words, it is sufficient in Eq. (3.11) to replace $\mu^{2}$ by $-\lambda^{2}$. The obtained expression is finite for all $\chi \in(0, \pi]$ and the averaged gravitational potential is equal to zero: $\bar{\varphi}=0 \Rightarrow$ $\overline{\delta \varepsilon}=0$.

\subsection{Hyperbolic space: $\mathcal{K}=-1$}

Here, the most interesting case corresponds to $\lambda^{2}=$ $\frac{8 \pi G_{\mathrm{N}}}{c^{4}} \varepsilon_{0} a_{0}^{2}+3>0$. This choice of sign gives a possibility to perform the transition to small values of the energy density of the perfect fluid: $\varepsilon_{0} \rightarrow 0$. Then the desired solution of Eq. (3.1) for a mass $m_{i}$ is

$\phi_{i}=-\frac{G_{\mathrm{N}} m_{i}}{\sinh \chi} \exp \left(-\chi \sqrt{\lambda^{2}+1}\right), \quad 0<\chi<+\infty$.

If the perfect fluid is absent $\left(\varepsilon_{0}=0\right)$, then we reproduce the formula obtained in [23]. On the other hand, the expression (3.12) shows that for $\varepsilon_{0}>0 \rightarrow \lambda^{2}+1>4$, the perfect fluid enhances the screening of the gravitating mass. For a many-particle system, the total gravitational potential takes the form

$\varphi=-G_{\mathrm{N}} \sum_{i} m_{i} \frac{\exp \left(-l_{i} \sqrt{\lambda^{2}+1}\right)}{\sinh l_{i}}+\frac{4 \pi G_{\mathrm{N}} \bar{\rho}}{\lambda^{2}}$,

where $l_{i}$ denotes the geodesic distance between the $i$ th mass $m_{i}$ and the point of observation. Similarly, using Eq. (3.11), we can write the expression for the total potential in the case of the spherical space.

Taking into account that the averaged total truncated potential has again the form (3.10), the procedure of averaging leads to the physically reasonable result: $\bar{\varphi}=0 \Rightarrow$ $\overline{\delta \varepsilon}=0$.

Concerning the case $\lambda^{2}<0$, the truncated gravitational potential is finite in the limit $\chi \rightarrow+\infty$. However, the procedure of averaging does not exist here. Therefore, this case is not of interest for us.

To conclude this section, we discuss briefly the case $\lambda^{2}=$ 0 . For $\mathcal{K}=0,-1$, the principle of superposition is absent now. To make the gravitational potential finite at any point including the spatial infinity, we need to cutoff it smoothly at some distances from each gravitating mass. If $\mathcal{K}=0$, then the perfect fluid is absent and this case was described in detail in [23]. It was shown that the averaged gravitational potential is not equal to zero. This is a disadvantage of such models. In the case $\mathcal{K}=+1$, the principle of superposition can be introduced due to the finiteness of the total volume of the Universe. Here, the comoving averaged rest mass density can be split as follows: $\bar{\rho}=\sum_{i} m_{i} /\left(2 \pi^{2}\right) \equiv \sum_{i} \bar{\rho}_{i}$. Then Eq. (2.25) can be solved separately for each combination $\left(m_{i}, \bar{\rho}_{i}\right)$. As a result, the gravitational potential of the $i$ th mass is

$\varphi_{i}=\frac{G_{\mathrm{N}} m_{i}}{2 \pi}-G_{\mathrm{N}} m_{i} \frac{\cos \chi}{\sin \chi}\left(1-\frac{\chi}{\pi}\right), \quad 0<\chi \leq \pi$.

This potential is convergent at any point $\chi \neq 0$, including $\chi=\pi$. It is not difficult to see that $\bar{\varphi}_{i}=0$. Therefore, the total averaged gravitational potential is also equal to zero: $\bar{\varphi}=\sum_{i} \bar{\varphi}_{i}=0 \Rightarrow \overline{\delta \varepsilon}=0$.

\section{Conclusion}

In our paper, we have considered the perfect fluids with the constant negative parameter $\omega$ of the EoS. We have investigated the role of these fluids for the Universe at late stages of its evolution. Such perfect fluids can be simulated by scalar fields with the corresponding form of the potentials $[13,14]$ as well as by the frustrated network of the topological defects [14,16-18]. Scalar fields with $-1<\omega<0$ and $\omega<-1$ are usually called quintessence and phantom, respectively, and they can be an alternative to the cosmological constant explaining the late time acceleration of the Universe. It takes place if their parameter of the $\operatorname{EoS} \omega<-1 / 3$. On the other hand, a small contribution from these fluids (e.g., the frustrated network of cosmic strings with $\omega=-1 / 3$ ) can explain the possible small departure from $\Lambda \mathrm{CDM}$ evolution [18].

To check the compatibility of these fluids with observations, we considered the present Universe at scales much less than the cell of homogeneity size which is approximately 190 Mpc [22]. At such distances, our Universe is highly inhomogeneous and the averaged Friedmann approach does not work here. We need to take into account the inhomogeneities in the form of galaxies, groups and clusters of galaxies. It is natural to assume also that the perfect fluid fluctuates around its average value. Therefore, these fluctuations as well as inhomogeneities perturb the FRW metrics. To consider these perturbations inside the cell of uniformity, we need to use the mechanical approach. This approach was established in our papers $[22,23]$. This is the novelty of our present work because in the previous studies the scalar perturbations were considered in the hydrodynamical approach which works well for the early Universe. It is obvious that the cosmological models should be consistent with the observations at 
all stages of the evolution of the Universe (both early and late).

Taking into account that the perturbations of the considered perfect fluids are purely adiabatic (i.e. dissipative processes are absent), we have shown that such perfect fluids are compatible with the theory of scalar perturbations if they satisfy two conditions. First, these fluids must be clustered (i.e. inhomogeneous). Second, the parameter of the EoS $\omega$ should be $-1 / 3$. Therefore, this perfect fluid neither accelerates nor decelerates the Universe. The frustrated network of the cosmic strings can be a candidate for this fluid. On the other hand, frustrated domain walls are ruled out because they have ${ }^{6} \omega=-2 / 3$.

Therefore, in the case of negative constant $\omega$, only models with $\omega=-1$ (a pure cosmological constant) and $\omega=-1 / 3$ are compatible with the mechanical approach, which is the most appropriate to describe the late Universe inside the cell of uniformity. Substituting $\omega=-1 / 3$ into the background Eq. (2.6), we can see that such perfect fluid behaves here as curvature. Hence, we can combine both terms to get a total "curvature" density parameter $\Omega_{\mathcal{K} \text {,tot }} \equiv \Omega_{\mathcal{K}}+\Omega_{\text {pf }}$. It is tempting to use the experimental restrictions on the curvature density parameter (see, e.g., sections 4.3 and 6.2.3 in [4] and [6], respectively) applying them for $\Omega_{\mathcal{K} \text {,tot }}$ and then to get limitations for $\lambda$ from Eq. (3.3). Exactly this parameter $\lambda$ determines the characteristic scales of the Yukawa-type screening in Eqs. (3.6) and (3.12). However, we cannot do it because the experimental restrictions have topological origin (i.e. they are due to the different form of the function $\Sigma$ in (2.2)) but not due to the fact that the curvature term in the Friedmann equations behaves as $1 / a^{2}$. In other words, the topological restrictions follow from the different definitions for the distances in the case of different topologies.

Then we have obtained the equation for the nonrelativistic gravitational potential. We need to know the form of the gravitational potential to describe dynamics of inhomogeneities. For example, all numerical simulations use the expression for the gravitational potentials of the inhomogeneities. Obviously, dynamical behavior of these inhomogeneities is determined by two competing mechanisms. On the one hand, it is the gravitational interaction between the inhomogeneities, and, on the other hand, the cosmological accelerated expansion. Therefore, one of the main tasks of the present paper was to study a possibility to get a reasonable form of the gravitational potential in the considered model. We have shown that due to the perfect fluid with $\omega=-1 / 3$, the physically reasonable solutions of the equation for the gravitational potential take place for flat, open, and closed Universes. The presence of this perfect fluid helps to resolve the Seeliger paradox [44]

\footnotetext{
6 This result may change if we take into account the shear deformations of the perfect fluid. However, this problem is out of the scope of our model and requires a separate investigation.
}

for any sign of the spatial curvature parameter $\mathcal{K}$. If the perfect fluid is absent, the hyperbolic space is preferred [23]. Hence, such perfect fluid can play an important role. This perfect fluid is concentrated around the inhomogeneities and results in screening of the gravitational potential. It should be noted that the obtained gravitational potentials have an important property: the total gravitational potentials averaged over the whole Universe are equal to zero $\bar{\varphi}=0$. Because the perfect fluid energy density fluctuation is proportional to the total gravitational potential $\delta \varepsilon \sim \varphi$, then the averaged energy density fluctuation is also equal to zero $\overline{\delta \varepsilon}=0$. Therefore, we arrive at the natural condition that the total perfect fluid energy density $\varepsilon=\bar{\varepsilon}+\delta \varepsilon$ after the procedure of the averaging is equal to $\bar{\varepsilon}$.

It must be emphasized that the case of imperfect fluids with the varying parameter $\omega$ (e.g., scalar fields with arbitrary potentials) requires a separate consideration which may lead to quite different conclusions. We intend to investigate this case in our forthcoming paper.

Acknowledgments The work of M. Eingorn was supported by NSF CREST award HRD-1345219 and NASA Grant NNX09AV07A.

Open Access This article is distributed under the terms of the Creative Commons Attribution License which permits any use, distribution, and reproduction in any medium, provided the original author(s) and the source are credited.

Funded by $\mathrm{SCOAP}^{3}$ / License Version CC BY 4.0.

\section{References}

1. A.G. Riess et al., Observational evidence from supernovae for an accelerating Universe and a cosmological constant. Astron. J. 116, 1009 (1998). arXiv:astro-ph/9805201

2. S. Perlmutter et al., Measurements of Omega and Lambda from 42 high-redshift supernovae. Astrophys. J. 517, 565 (1999). arXiv:astro-ph/9812133

3. M. Trodden, Dark energy and cosmology. arXiv:1212.6399 [astro$\mathrm{ph}]$

4. E. Komatsu et al., Seven-year Wilkinson Microwave Anisotropy Probe (WMAP) observations: cosmological interpretation. Astrophys. J. Suppl. 192, 18 (2011). arXiv:1001.4538 [astro-ph]

5. G. Hinshaw et al., Nine-year Wilkinson Microwave Anisotropy Probe (WMAP) observations: cosmological parameter results. arXiv:1212.5226 [astro-ph]

6. Planck Collaboration, Planck 2013 results. XVI. Cosmological parameters. arXiv:1303.5076 [astro-ph]

7. A.D. Dolgov, Cosmic antigravity. arXiv:1206.3725 [astro-ph]

8. P.G. Ferreira, M. Joyce, Structure formation with a selftuning scalar field. Phys. Rev. Lett. 79, 4740 (1997). arXiv:astro-ph/9707286

9. L. Wang, P.J. Steinhardt, Cluster abundance constraints for cosmological models with a timevarying, spatially inhomogeneous energy component with negative pressure. Astrophys. J. 508, 483 (1998). arXiv:astro-ph/9804015

10. I. Zlatev, L. Wang, P.J. Steinhardt, Quintessence, cosmic coincidence, and the cosmological constant. Phys. Rev. Lett. 82, 896 (1999). arXiv:astro-ph/9807002 
11. R.R. Caldwell, A phantom menace? Cosmological consequences of a dark energy component with super-negative equation of state. Phys. Lett. B 545, 23 (2002). arXiv:astro-ph/9908168

12. S.M. Carroll, M. Hoffman, M. Trodden, Can the dark energy equation-of-state parameter w be less than -1 ? Phys. Rev. D 68, 023509 (2003). arXiv:astro-ph/0301273

13. A. Zhuk, Integrable scalar field multi-dimensional cosmologies. Class. Quantum Gravity 13, 2163 (1996)

14. M. Bouhmadi-Lopez, P.F. Gonzalez-Diaz, A. Zhuk, Topological defect brane-world models. Gravit. Cosmol. 8, 285 (2002). arXiv:hep-th/0207170

15. J. Weller, A.M. Lewis, Large scale cosmic microwave background anisotropies and dark energy. Mon. Not. R. Astron. Soc. 346, 987 (2003). arXiv:astro-ph/0307104

16. A. Vilenkin, E.P.S. Shellard, Cosmic Strings and Other Topological Defects (Cambridge University Press, Cambridge, 1994)

17. P.P. Avelino, C.J.A.P. Martins, J. Menezes, R. Menezes, J.C.R.E. Oliveira, Scaling of cosmological domain wall networks with junctions. Phys. Lett. B 647, 63 (2007). arXiv:astro-ph/0612444

18. S. Kumar, A. Nautiyal, A.A. Sen, Deviation from $\Lambda$ CDM with cosmic strings networks. arXiv:1207.4024 [astro-ph]

19. O. Sergijenko, B. Novosyadlyj, Perturbed dark energy: classical scalar field versus tachyon. Phys. Rev. D 80, 083007 (2009). arXiv:0904.1583 [astro-ph]

20. M. Kunz, D. Sapone, Crossing the phantom divide. Phys. Rev. D 74, 123503 (2006). arXiv:astro-ph/0609040

21. O. Sergijenko, R. Durrer, B. Novosyadlyj, Observational constraints on scalar field models of dark energy with barotropic equation of state. JCAP 08, 004 (2011). arXiv:1102.3168 [astro-ph]

22. M. Eingorn, A. Zhuk, Remarks on mechanical approach to observable Universe. JCAP 05, 024 (2014). arXiv:1309.4924 [astro-ph]

23. M. Eingorn, A. Zhuk, Hubble flows and gravitational potentials in observable Universe. JCAP 09, 026 (2012). arXiv:1205.2384 [astro-ph]

24. J. Adamek, R. Durrer, M. Kunz, N-body methods for relativistic cosmology. arXiv:1408.3352 [astro-ph]

25. G.F.R. Ellis, G.W. Gibbons, Discrete Newtonian cosmology: perturbations. arXiv: 1409.0395 [gr-qc]

26. M. Eingorn, J. Novák, A. Zhuk, $f(R)$ gravity: scalar perturbations in the late Universe. Eur. Phys. J. C 74, 3005 (2014). arXiv:1401.5410 [astro-ph]

27. M. Brilenkov, M. Eingorn, L. Jenkovszky, A. Zhuk, Scalar perturbations in cosmological models with quark nuggets. Eur. Phys. J. C 74, 3011 (2014). arXiv:1310.4540 [astro-ph]

28. L.D. Landau, E.M. Lifshitz, in The classical Theory of Fields. Course of Theoretical Physics Series, vol. 2, 4th edn. (Oxford Pergamon Press, Oxford, 2000)
29. M. Eingorn, A. Kudinova, A. Zhuk, Dynamics of astrophysical objects against the cosmological background. JCAP 04, 010 (2013). arXiv:1211.4045 [astro-ph]

30. R. Bean, O. Dore, Probing dark energy perturbations: the dark energy equation of state and speed of sound as measured by WMAP. Phys. Rev. D 69, 083503 (2004). arXiv:astro-ph/0307100

31. V.F. Mukhanov, H.A. Feldman, R.H. Brandenberger, Theory of cosmological perturbations. Phys. Rep. 215, 203 (1992)

32. D.S. Gorbunov, V.A. Rubakov, Introduction to the Theory of the Early Universe: Cosmological Perturbations and Inflationary Theory (World Scientific, Singapore, 2011)

33. M. Eingorn, A. Zhuk, Classical tests of multidimensional gravity: negative result. Class. Quantum Gravity 27, 205014 (2010). arXiv: 1003.5690 [gr-qc]

34. W. Hu, Structure formation with generalized dark matter. Astrophys. J. 506, 485 (1998). arXiv:astro-ph/9801234

35. J.K. Erickson, R.R. Caldwell, P.J. Steinhardt, C. ArmendarizPicon, V. Mukhanov, Measuring the speed of sound of quintessence. Phys. Rev. Lett. 88, 121301 (2002). arXiv:astro-ph/0112438

36. J.-Q. Xia, Y.-F. Cai, T.-T. Qiu, G.-B. Zhao, X. Zhang, Constraints on the sound speed of dynamical dark energy. Int. J. Mod. Phys. D 17, 1229 (2008). arXiv:astro-ph/0703202

37. Y.-F. Cai, E.N. Saridakis, M.R. Setare, J.-Q. Xia, Quintom cosmology: theoretical implications and observations. Phys. Rep. 493, 1 (2010). arXiv:0909.2776 [hep-th]

38. P.J. Steinhardt, L. Wang, I. Zlatev, Cosmological tracking solutions. Phys. Rev. D 59, 1123504 (1999). arXiv:astro-ph/9812313

39. M. Bucher, D.N. Spergel, Is the dark matter a solid? Phys. Rev. D 60, 043505 (1999). arXiv:astro-ph/9812022

40. R.A. Battye, B. Carter, E. Chachoua, A. Moss, Rigidity and stability of cold dark solid universe model. Phys. Rev. D 72, 023503 (2005). arXiv:hep-th/0501244

41. L. Conversi, A. Melchiorri, L. Mersini, J. Silk, Are domain walls ruled out? Astropart. Phys. 21, 443 (2004). arXiv:astro-ph/0402529

42. P.J.E. Peebles, The Large-Scale Structure of the Universe (Princeton University Press, Princeton, 1980)

43. V. Springel, The cosmological simulation code GADGET-2. Mon. Not. R. Astron. Soc. 364, 1105 (2005)

44. J.D. Norton, The Cosmological Woes of Newtonian Gravitation Theory, in The Expanding Worlds of General Relativity: Einstein Studies, vol. 7, ed. by H. Goenner, J. Renn, J. Ritter, T. Sauer (Birkhauser, Boston, 1999), pp. 271-323 